\title{
Generalized set-theoretic unknown input observer for LPV systems with application to state estimation and robust fault detection
}

\author{
Feng Xu1 ${ }^{1}$, Junbo Tan ${ }^{1}$, Xueqian Wang ${ }^{1, *, \dagger}$, Vicenç Puig ${ }^{2}$, Bin Liang ${ }^{3, *, \dagger}$, Bo Yuan ${ }^{4}$ \\ and Houde $\mathrm{Liu}^{1}$ \\ ${ }^{1}$ Center of Intelligent Control and Telescience, Graduate School at Shenzhen, Tsinghua University, Shenzhen, China \\ ${ }^{2}$ Institut de Robòtica i Informàtica Industrial (CSIC-UPC), Technical University of Catalonia (UPC), Barcelona, Spain \\ ${ }^{3}$ Tsinghua National Laboratory for Information Science and Technology, Tsinghua University, Beijing, China \\ ${ }^{4}$ Intelligent Computing Lab, Graduate School at Shenzhen, Tsinghua University, Shenzhen, China
}

\begin{abstract}
SUMMARY
This paper proposes to design an unknown input observer (UIO) for the linear-parameter-varying (LPV) system on the basis of the set theory, which is named as the set-theoretic UIO (SUIO). The advantage of the SUIO consists in that it combines active and passive approaches to obtain robustness in state estimation (SE) and fault detection (FD). The active approach is based on the use of UIO to decouple unknown inputs, while the passive approach is based on the set theory to bound uncertain factors that cannot be actively decoupled. As a result, the effect of both unknown inputs (process disturbances, modeling errors, etc.) and measurement noises can be appropriately handled in the residual signals compared with the standard UIO-based SE and FD approaches. The design of SUIO can overcome the limitations of the traditional UIO design conditions, which can significantly broaden the application of the UIO-based SE and FD theory. Moreover, this paper proposes a generalized framework that can provide more flexibility in the design of SUIO guaranteeing their stability by means of a group of matrix inequalities. Because the LPV system uses a collection of online obtainable scheduling variables to embed nonlinearities, the design of SUIO for the LPV system can be used to address the SE and FD problems of nonlinear systems. At the end of this paper, two case studies are used to illustrate the effectiveness of the proposed approach. Copyright (C) 2017 John Wiley \& Sons, Ltd.
\end{abstract}

KEY WORDS: fault detection; state estimation; unknown input observer; set theory; LPV system

\section{INTRODUCTION}

For an fault detection (FD) approach, one of the most important features in practical applications is its robustness. In the literature, there mainly exist two families of approaches to obtain robustness in FD: active and passive [1,2]. The former decouples unknown inputs by generating residual signals that are insensitive to unknown inputs while maintaining the fault sensitivity. A well-known active decoupling approach is based on the use of unknown input observer (UIO). The latter obtains robustness by considering and propagating the bounds of unknown inputs, parametric uncertainties, and measurement noises to generate adaptive thresholds for residual signals. In this way, the effect of uncertainties on the residual is considered in a passive way without actively decoupling them as in the active approaches. The propagation of the uncertain factors is based on the use of the set theory, and the residual sets are described by using different geometric features such as polytopes,

\footnotetext{
*Correspondence to: Xueqian Wang, Center of Intelligent Control and Telescience, Graduate School at Shenzhen, Tsinghua University, Shenzhen, China and Bin Liang, Tsinghua National Laboratory for Information Science and Technology, Tsinghua University, Beijing, China.

${ }^{\dagger}$ E-mail: wang.xq@sz.tsinghua.edu.cn; bliang@tsinghua.edu.cn
} 
zonotopes, and ellipsoids (see [2-4] for more details on the set theory and their applications to robust fault diagnosis).

The UIO-based approaches have attracted considerable attention of researchers in recent decades because they can be designed to be insensitive to unknown inputs of a system [5]. As a result, the UIO-based approaches can decouple the effect of the unknown inputs on residual signals from that of faults. Thus, for a system, if such a UIO exists, it means that we can achieve robustness with respect to the unknown inputs. However, in order to design such a UIO, the system has to satisfy the matrix rank-based conditions of the standard UIO as given in [5], which could be difficult to be satisfied when there exists a large number of unknown inputs in the actual system. Thus, if for a given system that cannot satisfy the UIO design conditions, the objective to achieve FD robustness by using standard UIO-based approaches would be impossible. In this paper, in order to deal with this problem, we propose to divide unknown inputs into three groups. The first group of unknown inputs are actively decoupled by designing a UIO because these unknown inputs are selected to satisfy the UIO design conditions. The other two groups of unknown inputs cannot be decoupled from residual signals. Thus, we propose to use the set theory to deal with these unknown inputs by considering and propagating their bounds to generate adaptive residual thresholds. Then, robust FD decisions are made by testing whether the residuals obtained in real time are inside their thresholds or not. In this way, the unknown inputs that cannot be actively decoupled can be robustly handled in a passive way. Moreover, the obtained UIO integrating the set-theoretic methods (i.e., set-theoretic UIO (SUIO)) can not only be designed but also be robust to all unknown inputs.

In the literature, there are some works dealing with the design of UIO. In [5-7], different approaches were proposed to design the UIOs, and the corresponding design conditions were given. In [8], the classical interval observer-based FD approach was applied to the linear-parameter-varying (LPV) system by using linear matrix inequalities (LMIs) and zonotopes. In [9], the parity-space approach was extended for FD of the LPV system under the effect of unknown inputs. In [10], a different design approach of UIOs for the LPV systems was proposed, which was based on the assumption that the relative degree between outputs and unknown inputs was known. In [11], the authors proposed to design UIOs by dividing unknown inputs into two groups and decoupling part of them. However, the idea of handling the unknown inputs unable to actively be decoupled was based on a group of LMI conditions more difficult to be satisfied than that of the proposed set-theoretic approach. In [12], a set-based method was proposed for the UIO-based passive FD, which can only be used for the systems whose parametric matrices satisfy that the matrix $C E$ is invertible. Although there still exist other design methods of UIOs to relax the traditional design conditions in [5], such as the approaches proposed in $[13,14]$, those methods generally assume that either unknown inputs have mathematical relations with system states or the state-space description of unknown inputs is known, which in many cases could be far away from the reality. Other design approaches of UIOs were developed for linear time-delay systems, nonlinear systems, and switched linear systems [15-17]. Additionally, although this paper only focuses on robust SE and FD in the context of set-based and UIO-based approaches, there also exist some other robust FD approaches using different techniques and owning different advantages and disadvantages such as the frequency-domain techniques and some other observer-based techniques, among others (see [18-20]).

It should be emphasized that the SUIO can robustly handle the measurement noises together with the three groups of unknown inputs by using their bounds and the UIO, combining the advantages of the active and passive decoupling approaches and mitigating their disadvantages. Moreover, to the best knowledge of the authors, this feature is new because current works on the UIO-based FD either do not consider the measurement noises or assume that the measurement noises have known mathematical relations with the unknown inputs, which are far away from the reality, and means that the existent design methods of UIO have difficulties to cope with the measurement noises. Moreover, we design SUIOs for the LPV systems to deal with the existence of nonlinearities in actual systems. In this way, we will be able to broaden the applicability and benefit from the advantages of SUIOs in SE and FD applications.

The current paper is based on preliminary results by authors recently presented in [21, 22]. In [22], a preliminary algorithm combining active and passive robust approaches was presented, and 
[22] used the set-membership-estimation framework and the UIO for robust estimation of state sets. Based on these preliminary results, the current work aims to present deeper results on robust SE and FD applications of SUIO and the extension to the LPV systems. Thus, the main motivations and contributions of this paper are in the following:

- The standard design conditions of UIOs can be effectively relaxed by dividing all unknown inputs into several groups. The UIO is only designed to actively decouple part of the unknown inputs, which satisfy the design conditions of the UIOs.

- The remaining unknown inputs that cannot be decoupled by the UIO and the measurement noises can be handled by using the set-theoretic approaches with only considering their bounds, extending the applicability of available UIO-based FD approaches.

- The SE and FD conservatism of the standard set-based approaches can be reduced because part of the unknown inputs will be decoupled by the UIO and only the non-decoupled unknown inputs and noises will be propagated generating bounds for states, outputs, and residuals.

- A generalized SUIO structure mixing the traditional UIO framework with their SE-error dynamics is proposed in order to obtain more design degrees of freedom of parametric matrices of SUIO, which provides a new perspective of observer design for robust SE and FD.

The remainder of this paper is as follows. Section 2 introduces the LPV model and the standard design of UIO. Section 3 proposes the design of SUIO for the LPV system. Section 4 proposes the SUIO-based robust SE and FD approaches. The effectiveness of this approach is illustrated in Section 5. Finally, the paper is concluded in Section 6.

\section{PROBLEM FORMULATION}

\subsection{System description}

In this paper, we consider the following discrete-time LPV model:

$$
\begin{gathered}
x_{k+1}=A\left(\rho_{k}\right) x_{k}+B\left(\rho_{k}\right) u_{k}+E\left(\rho_{k}\right) \omega_{k}, \\
y_{k}=C x_{k}+F \eta_{k},
\end{gathered}
$$

where $A\left(\rho_{k}\right) \in \mathbb{R}^{n \times n}, B\left(\rho_{k}\right) \in \mathbb{R}^{n \times p}, E\left(\rho_{k}\right) \in \mathbb{R}^{n \times r}$ are parametric matrices dependent on a scheduling vector $\rho_{k} \in \mathbb{R}^{m} ; C \in \mathbb{R}^{q \times n}$ and $F \in \mathbb{R}^{q \times s}$ are constant matrices; $k$ denotes the $k$-th discrete time instant; $x_{k} \in \mathbb{R}^{n}$ and $y_{k} \in \mathbb{R}^{q}$ denote the state and output vectors; $u_{k} \in \mathbb{R}^{p}$ and $\omega_{k} \in \mathbb{R}^{r}$ represent the known and unknown inputs (i.e., process disturbances and modeling errors), respectively; and $\eta_{k} \in \mathbb{R}^{s}$ represents the measurement noise vector.

It is assumed that the scheduling vector $\rho_{k}$ is composed of $m$ obtainable variables as

$$
\rho_{k}=\left[\begin{array}{llll}
\rho_{1, k} & \rho_{2, k} & \cdots & \rho_{m, k}
\end{array}\right],
$$

and that $\rho_{k}$ is bounded by a polytopic set as

$$
\rho_{k} \in \mathcal{P},
$$

where

$$
\begin{aligned}
\mathcal{P} & =\left[\begin{array}{llll}
P_{1} & P_{2} & \ldots & P_{m}
\end{array}\right]^{T}, \\
P_{i} & =\left[\begin{array}{ll}
\rho_{i, k}, \bar{\rho}_{i, k}
\end{array}\right], \\
\rho_{i, k} & \in P_{i} \text { for } i=1,2, \ldots, m .
\end{aligned}
$$

Remark 2.1

$\mathcal{P}$ is typically defined as an interval vector (i.e., a box) composed of the intervals of the components of $\rho_{k}\left(P_{i}\right.$ is the interval for the $i$-th component of $\rho_{k}$, and $\underline{\rho}_{i, k}$ and $\bar{\rho}_{i, k}$ are the minimal and maximal bounds of $\rho_{i, k}$, respectively). 


\subsection{UIO for LPV system}

According to [10, 22], a UIO for the LPV system (1) can be designed as

$$
\begin{gathered}
z_{k+1}=N\left(\rho_{k}\right) z_{k}+T\left(\rho_{k}\right) u_{k}+K\left(\rho_{k}\right) y_{k}, \\
\hat{x}_{k}=M z_{k}+H y_{k}, \\
\hat{y}_{k}=C \hat{x}_{k},
\end{gathered}
$$

where $N\left(\rho_{k}\right), T\left(\rho_{k}\right), K\left(\rho_{k}\right), M$, and $H$ are the parametric matrices of the UIO, which have compatible dimensions; $z_{k}$ is the state vector of the UIO; and $\hat{x}_{k}$ and $\hat{y}_{k}$ are the estimated state and output vectors, respectively. Based on (1) and (3), we can define the SE-error vector

$$
e_{k}^{x}=x_{k}-\hat{x}_{k},
$$

whose dynamics can be derived as

$$
\begin{aligned}
e_{k+1}^{x}= & \left(A\left(\rho_{k}\right)-H C A\left(\rho_{k}\right)-M K_{1}\left(\rho_{k}\right) C\right) e_{k}^{x} \\
& +\left[\left(A\left(\rho_{k}\right)-H C A\left(\rho_{k}\right)-M K_{1}\left(\rho_{k}\right) C\right) M-M N\left(\rho_{k}\right)\right] z_{k} \\
& +\left[\left(A\left(\rho_{k}\right)-H C A\left(\rho_{k}\right)-M K_{1}\left(\rho_{k}\right) C\right) H-M K_{2}\left(\rho_{k}\right)\right] y_{k} \\
& +\left(B\left(\rho_{k}\right)-M T\left(\rho_{k}\right)-H C B\left(\rho_{k}\right)\right) u_{k}+\left(E\left(\rho_{k}\right)-H C E\left(\rho_{k}\right)\right) \omega_{k} \\
& -H F \eta_{k+1}-M K_{1}\left(\rho_{k}\right) F \eta_{k} .
\end{aligned}
$$

The decomposition of the parametric matric $K\left(\rho_{k}\right)$ into another two matrices as

$$
K\left(\rho_{k}\right)=K_{1}\left(\rho_{k}\right)+K_{2}\left(\rho_{k}\right),
$$

can help us obtain the terms $\left(A\left(\rho_{k}\right)-H C A\left(\rho_{k}\right)-M K_{1}\left(\rho_{k}\right) C\right) e_{k}^{x},\left[\left(A\left(\rho_{k}\right)-H C A\left(\rho_{k}\right)-\right.\right.$ $\left.\left.M K_{1}\left(\rho_{k}\right) C\right) M-M N\left(\rho_{k}\right)\right] z_{k}$, and $\left[\left(A\left(\rho_{k}\right)-H C A\left(\rho_{k}\right)-M K_{1}\left(\rho_{k}\right) C\right) H-M K_{2}\left(\rho_{k}\right)\right] y_{k}$ required for the design of the UIO ((6a), (6b), and (6c)). This way of derivation can be widely found in the literature on design of UIO [5]. Thus, from (5), the parametric matrices can be obtained

$$
\begin{gathered}
\Lambda_{0}\left(\rho_{k}\right)=A\left(\rho_{k}\right)-H C A\left(\rho_{k}\right)-M K_{1}\left(\rho_{k}\right) C, \\
\Lambda_{1}\left(\rho_{k}\right)=\left(A\left(\rho_{k}\right)-H C A\left(\rho_{k}\right)-M K_{1}\left(\rho_{k}\right) C\right) M-M N\left(\rho_{k}\right), \\
\Lambda_{2}\left(\rho_{k}\right)=\left(A\left(\rho_{k}\right)-H C A\left(\rho_{k}\right)-M K_{1}\left(\rho_{k}\right) C\right) H-M K_{2}\left(\rho_{k}\right), \\
\Lambda_{3}\left(\rho_{k}\right)=B\left(\rho_{k}\right)-M T\left(\rho_{k}\right)-H C B\left(\rho_{k}\right), \\
\Lambda_{4}\left(\rho_{k}\right)=E\left(\rho_{k}\right)-H C E\left(\rho_{k}\right), \\
\Lambda_{5}\left(\rho_{k}\right)=-H F, \\
\Lambda_{6}\left(\rho_{k}\right)=-M K_{1}\left(\rho_{k}\right) F .
\end{gathered}
$$

Provided that the standard method of designing UIOs, such as the one proposed in [5], is followed to design the UIO for the LPV system based on (6), the following conditions:

$$
\Lambda_{1}\left(\rho_{k}\right)=0,
$$




$$
\begin{aligned}
& \Lambda_{2}\left(\rho_{k}\right)=0, \\
& \Lambda_{3}\left(\rho_{k}\right)=0, \\
& \Lambda_{4}\left(\rho_{k}\right)=0
\end{aligned}
$$

should simultaneously hold. Note that, in order to decouple the effect of $\omega_{k}$ on the SE-error vector, we must guarantee that (7d) can be solved. According to the results in [5], a sufficient and necessary condition to guarantee the solvability of $(7 \mathrm{~d})$ is given as

$$
\operatorname{rank}\left(E\left(\rho_{k}\right)\right)=\operatorname{rank}\left(C E\left(\rho_{k}\right)\right) \text {, for all } \rho_{k} \in \mathcal{P} .
$$

Remark 2.2

Under condition (7d), all the unknown inputs included in $\omega_{k}$ can be decoupled, provided that the matrix rank-based decoupling condition (8) is satisfied.

\section{Remark 2.3}

If we want to further decouple the effect of the measurement-noise vector $\eta_{k}$ on the SE-error vector, we have to additionally satisfy $\Lambda_{5}\left(\rho_{k}\right)=0$ and $\Lambda_{6}\left(\rho_{k}\right)=0$. By analyzing (6f) and (6g), it can be observed that it is not easy to decouple the measurement noise vectors $\eta_{k}$ and $\eta_{k+1}$ simultaneously under the conditions (7) by only using the standard UIO design method.

As a result, under the condition (7), the SE-error vector can be derived as

$$
e_{k+1}^{x}=\Lambda_{0}\left(\rho_{k}\right) e_{k}^{x}+\Lambda_{5}\left(\rho_{k}\right) \eta_{k+1}+\Lambda_{6}\left(\rho_{k}\right) \eta_{k},
$$

where, as aforementioned, the matrices $\Lambda_{0}\left(\rho_{k}\right)$ must be designed to stabilize the dynamics (9). In this way, the UIO for the LPV system can be obtained.

Note that, using the standard design approach of the UIO for the LPV system, we have to compute all the parametric matrices of the UIO (3) by solving the matrix equations (7) online. It is obvious that the computational complexity is high. In the following, we will propose a design approach on the basis of the polytopic form of the LPV system to reduce the implemental complexity of the UIO.

\subsection{Polytopic LPV system}

As defined in (2), the polytope $\mathcal{P}$ has $m$ components, which has $N=2^{m}$ vertices. Thus, for a polytopic LPV system, a matrix $J\left(\rho_{k}\right)$ parameterized by $\rho_{k}$ can be equivalently written into

$$
J\left(\rho_{k}\right)=\sum_{i=1}^{N} \lambda_{i}\left(\rho_{k}\right) J_{i}
$$

with $J_{i}$ is a constant matrix and

$$
\sum_{i=1}^{N} \lambda_{i}\left(\rho_{k}\right)=1, \quad 0 \leqslant \lambda_{i}\left(\rho_{k}\right) \leqslant 1,
$$

which means that $A\left(\rho_{k}\right), B\left(\rho_{k}\right), E\left(\rho_{k}\right), N\left(\rho_{k}\right), T\left(\rho_{k}\right)$, and $K\left(\rho_{k}\right)$ can be written into the same form as well. For simplicity, a set $\mathbb{I}=\{1,2, \ldots, N\}$ is defined for the index $i$ in (10) and (11).

Thus, on the basis of (10) and (11), the dynamics (5) can be equivalently transformed into

$$
\begin{aligned}
e_{k+1}^{x}= & \sum_{i=1}^{N} \lambda_{i}\left(\rho_{k}\right)\left\{\left(A_{i}-H C A_{i}-M K_{i, 1} C\right) e_{k}^{x}\right. \\
& +\left[\left(A_{i}-H C A_{i}-M K_{i, 1} C\right) M-M N_{i}\right] z_{k} \\
& +\left[\left(A_{i}-H C A_{i}-M K_{i, 1} C\right) H-M K_{i, 2}\right] y_{k} \\
& \left.+\left(B_{i}-M T_{i}-H C B_{i}\right) u_{k}+\left(E_{i}-H C E_{i}\right) \omega_{k}-M K_{i, 1} F \eta_{k}\right\} \\
& -H F \eta_{k+1},
\end{aligned}
$$


with

$$
K_{i}=K_{i, 1}+K_{i, 2} .
$$

Thus, the active decoupling condition (8) of UIO can be equivalently transformed into

$$
\sum_{i=1}^{N} \lambda_{i}\left(\rho_{k}\right) E_{i}=\sum_{i=1}^{N} \lambda_{i}\left(\rho_{k}\right) H C E_{i} .
$$

It can be observed that a group of sufficient conditions to guarantee (13) can be obtained as

$$
E_{i}=H C E_{i}, \text { for each } i \in \mathbb{I},
$$

where the necessary and sufficient condition to guarantee that (14) has solutions is given as

$$
\operatorname{rank}\left(C E_{i}\right)=\operatorname{rank}\left(E_{i}\right),
$$

for each group of vertices of the LPV system. This means that by designing the matrix $H$ to satisfy (15), the implementation of active decoupling of the unknown inputs for the LPV system can be simplified. Similarly, from (12), the following parametric matrices can be extracted as

$$
\begin{gathered}
\Lambda_{i, 0}=A_{i}-H C A_{i}-M K_{i, 1} C, \\
\Lambda_{i, 1}=\left(A_{i}-H C A_{i}-M K_{i, 1} C\right) M-M N_{i}, \\
\Lambda_{i, 2}=\left(A_{i}-H C A_{i}-M K_{i, 1} C\right) H-M K_{i, 2}, \\
\Lambda_{i, 3}=B_{i}-M T_{i}-H C B_{i}, \\
\Lambda_{i, 4}=E_{i}-H C E_{i}, \\
\Lambda_{i, 5}=-H F, \\
\Lambda_{i, 6}=-M K_{i, 1} F .
\end{gathered}
$$

such that according to the standard procedure, the UIO can be designed by satisfying

$$
\begin{aligned}
& \Lambda_{i, 1}=0, \\
& \Lambda_{i, 2}=0, \\
& \Lambda_{i, 3}=0, \\
& \Lambda_{i, 4}=0,
\end{aligned}
$$

where (17) is a sufficient condition to guarantee the satisfaction of (7).

\section{DESIGN OF SUIO FOR LPV SYSTEM}

In order to consider the effect of the measurement-noise vector $\eta_{k}$ on robust $\mathrm{FD}$, a new design method is proposed for the UIO by using the set theory in this section. By using the proposed method, on one hand, $\eta_{k}$ can be passively handled to achieve FD robustness. On the other hand, the satisfaction of the design conditions of the standard UIOs recalled in the previous section can be relaxed identifying a group of unknown inputs that can be decoupled. 


\subsection{Handling of unknown inputs}

As previously discussed, in order to decouple the unknown input vector $\omega_{k}$ by using the standard UIO, the matrix rank-based decoupling conditions (15) for all $i \in \mathbb{I}$ must be satisfied, which could be difficult to achieve when the number of unknown inputs is large. Thus, in order to deal with this problem, we propose to divide the unknown inputs into three groups, where only the first group includes the unknown inputs that can be actively decoupled. As a result, we have the following partitioning of the unknown-input vector:

$$
\omega_{k}=\left[\begin{array}{lll}
\left(\omega_{1, k}\right)^{T} & \left(\omega_{2, k}\right)^{T} & \left(\omega_{3, k}\right)^{T}
\end{array}\right]^{T},
$$

where $\omega_{1, k} \in \mathbb{R}^{n_{1}}, \omega_{2, k} \in \mathbb{R}^{n_{2}}$, and $\omega_{3, k} \in \mathbb{R}^{r-n_{1}-n_{2}} . \omega_{1, k}$ denotes the unknown inputs that can be actively decoupled because (15) for all $i \in \mathbb{I}$ is satisfied. On the other hand, $\omega_{2, k}$ includes the unknown inputs that satisfy (15) for only some $i \in \mathbb{I}$, while for some other $i \in \mathbb{I}$, they do not satisfy. Finally, $\omega_{3, k}$ denotes the unknown inputs that do not satisfy (15) for any $i \in \mathbb{I}$.

\section{Remark 3.1}

The $i$-th group of conditions aforementioned means that, for the design conditions (17), the index $i$ taking each value inside $\mathbb{I}$ corresponds to a group of the design conditions. Thus, we have $N$ groups of design conditions in total for a UIO based on the polytopic model of the LPV system, each corresponding to a group of vertices.

By taking (18) into account, the matrix $E_{i}$ should also be divided into

$$
E_{i}=\left[\begin{array}{lll}
E_{i, 1} & E_{i, 2} & E_{i, 3}
\end{array}\right],
$$

where $E_{i, 1} \in \mathbb{R}^{n \times n_{1}}, E_{i, 2} \in \mathbb{R}^{n \times n_{2}}$, and $E_{i, 3} \in \mathbb{R}^{n \times\left(r-n_{1}-n_{2}\right)}$.

\subsection{Design of SUIO}

By substituting (18) and (19) into (12), the dynamics of the SE-error vector can be rewritten as

$$
\begin{aligned}
e_{k+1}^{x}= & \sum_{i=1}^{N} \lambda_{i}\left(\rho_{k}\right)\left\{\Lambda_{i, 0} e_{k}^{x}+\Lambda_{i, 1} z_{k}+\Lambda_{i, 2} y_{k}+\Lambda_{i, 3} u_{k}+\Lambda_{i, 7} \omega_{1, k}\right. \\
& \left.+\Lambda_{i, 8} \omega_{2, k}+\Lambda_{i, 9} \omega_{3, k}+\Lambda_{i, 6} \eta_{k}\right\}+\Lambda_{i, 5} \eta_{k+1},
\end{aligned}
$$

where

$$
\begin{aligned}
& \Lambda_{i, 7}=E_{i, 1}-H C E_{i, 1}, \\
& \Lambda_{i, 8}=E_{i, 2}-H C E_{i, 2}, \\
& \Lambda_{i, 9}=E_{i, 3}-H C E_{i, 3} .
\end{aligned}
$$

Thus, if the matrix $\Lambda_{i, 7}$ can be designed to be a zero matrix, then the effect of $\omega^{1, k}$ on SE and FD can be decoupled, that is,

$$
\Lambda_{i, 7}=\mathbf{0},
$$

which is the basic idea of the proposed approach.

\section{Remark 3.2}

The dynamics (20) is a general form of the dynamics of the SE-error vector. This means that the number $n_{1}$ of columns of the matrix $E_{i, 1}$ takes a value from the interval $[0, r]$ where $n_{1}=0$ implies that it is impossible to decouple any unknown input only based on the UIO structure (3), $n_{1}=r$ means that all the unknown inputs included in $\omega_{k}$ can be decoupled, while $n_{1}$ taking a value from the interval $(0, r)$ means that only part of unknown inputs (i.e., $\left.\omega_{1, k}\right)$ can be decoupled. 
Because the proposed approach aims to deal with the systems whose unknown inputs cannot be completely decoupled by the UIO, it is not required to satisfy (21b) and (21c) when the condition (22) is already satisfied in the current paper. Additionally, if the unknown inputs cannot be divided into several groups, the situation can be considered as two cases. The first one is that there does not exist one unknown input that can be actively decoupled by the SUIO. In this case, we could passively decouple the effect of all the unknown inputs (i.e., the traditional set-based approaches). The other case is that all the unknown inputs can be actively decoupled by the SUIO. This case can be considered similar to the traditional UIO-based approaches.

\section{Remark 3.3}

Equation 22 is a condition that can be satisfied relatively easily because the worst case of $E_{i, 1}$ (i.e., $E_{i, 1}$ becomes a zero matrix) can always be a design choice. Thus, the difficulty of satisfying the UIO-designing condition (22) is much less than that of the condition (15). Similarly, the necessary and sufficient condition to solve (22) is a matrix rank-based condition

$$
\operatorname{rank}\left(C E_{i, 1}\right)=\operatorname{rank}\left(E_{i, 1}\right) \text {, }
$$

for all $i \in \mathbb{I}$. In general, (23) can always hold because $E_{i, 1}$ can take the zero matrix to satisfy (23).

Therefore, according to (17), the design conditions for the proposed UIO method turns into

$$
\begin{aligned}
& \Lambda_{i, 1}=0, \\
& \Lambda_{i, 2}=0, \\
& \Lambda_{i, 3}=0, \\
& \Lambda_{i, 7}=0 .
\end{aligned}
$$

\section{Remark 3.4}

The necessary and sufficient conditions for the existence of UIO satisfying (20) and (24) can be obtained by a straightforward extension of the corresponding design conditions of the traditional UIO in [5] to the case of (23).

Consequently, the corresponding dynamics of the SE-error vector is obtained as

$$
e_{k+1}^{x}=\sum_{i=1}^{N} \lambda_{i}\left(\rho_{k}\right)\left\{\Lambda_{i, 0} e_{k}^{x}+\Lambda_{i, 8} \omega_{2, k}+\Lambda_{i, 9} \omega_{3, k}+\Lambda_{i, 6} \eta_{k}\right\}+\Lambda_{i, 5} \eta_{k+1},
$$

where the matrices $\Lambda_{i, 0}$ for all $i \in \mathbb{I}$ should be designed to be stable by choosing proper parametric matrices. It can be seen that $\omega_{2, k}, \omega_{3, k}$, and $\eta_{k}$ can still affect the SE-error vector. This implies that, in order to obtain FD robustness, we have to further decouple their effect on residual signals. In this paper, we turn to the set theory to passively decouple them and to achieve FD robustness. Thus, it is assumed that $\omega_{2, k}, \omega_{3, k}$, and $\eta_{k}$ are bounded in known sets, that is,

$$
\begin{gathered}
W_{2}=\left\{\omega_{2} \in \mathbb{R}^{n_{2}}:\left|\omega_{2}-\omega_{2}^{c}\right| \leqslant \bar{\omega}_{2}\right\}, \\
W_{3}=\left\{\omega_{3} \in \mathbb{R}^{n_{3}}:\left|\omega_{3}-\omega_{3}^{c}\right| \leqslant \bar{\omega}_{3}\right\}, \\
V=\left\{\eta \in \mathbb{R}^{s}:\left|\eta-\eta^{c}\right| \leqslant \bar{\eta}\right\},
\end{gathered}
$$

where $\omega_{2}^{c}, \bar{\omega}_{2}, \omega_{3}^{c}, \bar{\omega}_{3}, \eta^{c}$, and $\bar{\eta}$ are constant vectors. By substituting $W_{2}, W_{3}$, and $V$ into (25) to replace $\omega_{2, k}, \omega_{3, k}, \eta_{k}$, and $\eta_{k+1}$, we can further obtain

$$
\begin{aligned}
\mathbb{E}_{k+1}^{x}= & \left.\left.\sum_{i=1}^{N} \lambda_{i}\left(\rho_{k}\right) \Lambda_{i, 0}\right) \mathbb{E}_{k}^{x} \oplus \sum_{i=1}^{N} \lambda_{i}\left(\rho_{k}\right) \Lambda_{i, 8}\right) W_{2} \\
& \left.\left.\oplus \sum_{i=1}^{N} \lambda_{i}\left(\rho_{k}\right) \Lambda_{i, 9}\right) W_{3} \oplus \sum_{i=1}^{N} \lambda_{i}\left(\rho_{k}\right) \Lambda_{i, 6}\right) V \oplus \Lambda_{i, 5} V,
\end{aligned}
$$


where $\mathbb{E}_{k}^{x}$ denotes the set of the SE-error vector $e_{k}$ at time instant $k$ and $\oplus$ denotes the Minkowski sum. Furthermore, we can obtain the output-estimation-error vector $e_{k}^{y}$ as

$$
e_{k}^{y}=y_{k}-\hat{y}_{k}=C e_{k}^{x}+F \eta_{k} .
$$

By taking the sets of $e_{k}^{x}$ and $\eta_{k}$ into account, the set $\mathbb{E}_{k}^{y}$ of the output-estimation-error vector $e_{k}^{y}$ at time instant $k$ can be obtained as

$$
\mathbb{E}_{k}^{y}=C \mathbb{E}_{k}^{x} \oplus F V .
$$

Thus, under the conditions (24), an SUIO can be constructed, that is, as a whole, the SUIO of the system (1) is composed of the Eqs (3), (27), and (29), which is robust to both the unknown inputs and the measurement noises when it is used for robust FD.

\section{ROBUST FAULT DETECTION AND STATE ESTIMATION}

This section will introduce the proposed SUIO-based FD approach for the LPV system (1).

\subsection{SUIO-based fault detection}

For the observer-based FD approaches, we should first define the residual signals for the implementation of FD. In this paper, the output-estimation-error vector $e_{k}^{y}$ as in (28) is defined as the residual vector, which can be computed in real time.

For the proposed approach, equations (27) and (29) are used to generate the estimation sets $\mathbb{E}_{k}^{x}$ and $\mathbb{E}_{k}^{y}$ in real time. On the basis of (27) and (29), we should first make an assumption on its initial set and the initial condition for the system (1) as in Assumption 4.1.

\section{Assumption 4.1}

The initial set of the dynamics (27) and the initial SE-error vector are denoted as $\mathbb{E}_{0}^{x}$ and $e_{0}^{x}$, respectively, and the initial condition $e_{0}^{x} \in \mathbb{E}_{0}^{x}$ holds.

Under Assumption 4.1, as long as the system is in healthy operation, it can be guaranteed to have

$$
\begin{aligned}
& e_{k}^{x} \in \mathbb{E}_{k}^{x}, \\
& e_{k}^{y} \in \mathbb{E}_{k}^{y} .
\end{aligned}
$$

Because $e_{k}^{x}$ cannot be used for FD because the state is not accessible, the proposed SUIO uses (30b) as the FD criterion to judge whether the system has become faulty. Furthermore, an equivalent FD criterion of (30b) can be obtained as

$$
\mathbf{0} \in R_{k},
$$

where

$$
R_{k}=\left\{e_{k}^{y}\right\} \oplus\left(-\mathbb{E}_{k}^{y}\right)
$$

is named as the residual set in this paper. Thus, if (30b) or (31) is violated, we think that the system has become faulty. Otherwise, it is concluded that the system is still healthy.

\section{Remark 4.1}

It is possible that there exist some faults dependent of their sizes that do not violate the FD criterion (30b) or (31). These faults would not be detectable because they produce an effect on the residual smaller than the uncertain factors the system has. Thus, in such a situation, we assume that the system is healthy. 


\subsection{Zonotopic implementation of SUIO}

In the previous sections, the principle of the proposed SUIO-based approach have been explained. However, we should further indicate how to compute all relevant sets and implement the proposed SUIO-based approach. In the literature, there exist several geometric sets such as polytopes, ellipsoids, and zonotopes. Considering geometric features and computational complexity, zonotopes are chosen to implement the proposed SUIO-based method.

\section{Definition 4.1}

An $m$-order zonotope $Z$ is defined as $Z=g \oplus H \mathbb{B}^{m}$, where $g$ and $H$ are called the center and segment matrix, respectively, and $\mathbb{B}^{m}$ is an $m$-dimensional unitary box whose elements are the unitary intervals $[-1,1]$.

\section{Remark 4.2}

For simplicity, the notation of a zonotope $Z=g \oplus H \mathbb{B}^{m}$ is reduced to $Z(g, H)$ in the following contents of this paper.

For the implementation of the proposed approach, the following zonotope properties are recalled.

Property 4.1

Given two zonotopes $Z_{1}=Z\left(g_{1}, H_{1}\right)$ and $Z_{2}=Z\left(g_{2}, H_{2}\right), Z_{1} \oplus Z_{2}=Z\left(g_{1}+g_{2},\left[H_{1} H_{2}\right]\right)$.

\section{Property 4.2}

Given a zonotope $Z=Z(g, H)$ and a compatible matrix $K, K Z=Z(K g, K H)$.

Furthermore, we introduce the following notations:

$$
\begin{gathered}
W_{2}=Z\left(\omega_{2}^{c}, H_{\omega_{2}}\right), \\
W_{3}=Z\left(\omega_{3}^{c}, H_{\omega_{3}}\right), \\
V=Z\left(\eta^{c}, H_{\eta}\right), \\
\mathbb{E}_{k}^{x}=Z\left(e_{k}^{c, x}, H_{e_{k}^{x}}\right), \\
\mathbb{E}_{k}^{y}=Z\left(e_{k}^{c, y}, H_{e_{k}^{y}}\right) .
\end{gathered}
$$

\section{Remark 4.3}

According to (26), it is straightforward to rewrite $W_{2}, W_{3}$, and $V$ into zonotopic form.

According to (27) and (29), the centers and segment matrices of the estimated state and output zonotopes can be obtained by using Properties 4.1 and 4.2 as

$$
\begin{aligned}
& \left.e_{k+1}^{c, x}=\sum_{i=1}^{N} \lambda_{i}\left(\rho_{k}\right) \Lambda_{i, 0}\right) e_{k}^{c, x}+\left(\sum_{i=1}^{N} \lambda_{i}\left(\rho_{k}\right) \Lambda_{i, 8}\right) \omega_{2}^{c} \\
& \left.\left.+\sum_{i=1}^{N} \lambda_{i}\left(\rho_{k}\right) \Lambda_{i, 9}\right) \omega_{3}^{c}+\Lambda_{i, 5} \eta^{c}+\sum_{i=1}^{N} \lambda_{i}\left(\rho_{k}\right) \Lambda_{i, 6}\right) \eta^{c}, \\
& H_{e_{k+1}^{x}}=\left[\left(\sum_{i=1}^{N} \lambda_{i}\left(\rho_{k}\right) \Lambda_{i, 0}\right) H_{e_{k}^{x}} \quad \sum_{i=1}^{N} \lambda_{i}\left(\rho_{k}\right) \Lambda_{i, 8}\right) H_{\omega_{2}} \\
& \left.\left.\left.\sum_{i=1}^{N} \lambda_{i}\left(\rho_{k}\right) \Lambda_{i, 9}\right) H_{\omega_{3}} \Lambda_{i, 5} H_{\eta} \quad \sum_{i=1}^{N} \lambda_{i}\left(\rho_{k}\right) \Lambda_{i, 6}\right) H_{\eta}\right],
\end{aligned}
$$




$$
\begin{aligned}
& e_{k}^{c, y}=C e_{k}^{c, x}+F \eta^{c}, \\
& H_{e_{k}^{y}}=\left[\begin{array}{ll}
C H_{e_{k}^{x}} & F H_{\eta}
\end{array}\right] .
\end{aligned}
$$

Note that $\mathbb{E}_{k}^{x}$ and $\mathbb{E}_{k}^{y}$ can be obtained by indirectly computing their centers and segment matrices, respectively. Moreover, in real-time operation, the order of zonotopes should be controlled as well, which is based on considering Property 4.3.

Property 4.3

Given a zonotope $Z=g \oplus H \mathbb{B}^{m} \subset \mathbb{R}^{n}$ and an integer $s$ (with $n<s<m$ ), denote by $\hat{H}$ the matrix resulting from the reordering of the columns of the matrix $H$ in decreasing Euclidean norm. Then $Z \subseteq g \oplus\left[\hat{H}_{T} \quad Q\right] \mathbb{B}^{s}$ where $\hat{H}_{T}$ is obtained from the first $s-n$ columns of the matrix $\hat{H}$ and $Q \in \mathbb{R}^{n \times n}$ is a diagonal matrix whose elements satisfy $Q_{i i}=\sum_{j=s-n+1}^{m}\left|\hat{H}_{i j}\right|, i=1, \ldots, n$.

\subsection{Further extension of SUIO-based framework}

It has been shown that the proposed SUIO is less constrained than that of the traditional UIO because it only decouples those unknown inputs that satisfy the design conditions. However, the conditions in (24) have to be satisfied for all $i \in \mathbb{I}$, which constrain the design of parametric matrices. This section will propose methods to relax these restrictions.

4.3.1. Analysis of SUIO-based framework. Under the conditions in (24), the SE-error vector is subject to the dynamics (27). Because $\omega_{2, k}^{i}, \eta_{k+1}$ and $\eta_{k}$ are bounded as in (26), it can be concluded that the SE-error vector will converge to an invariant set. The notion of invariant sets is given in Definition 4.2.

\section{Definition 4.2}

A set $X$ is a robust positively invariant set of the dynamics $x_{k+1}=f\left(x_{k}, \omega_{k}\right)$ if for any $x_{k} \in X$ and $\omega_{k} \in W, x_{k+1} \in X$ is satisfied .

The SUIO is a state/output observer for the system (1) because the SE-error vector is free from the effect of $z_{k}, y_{k}$, and $u_{k}$ under the conditions (24a), (24b), and (24c). Actually, it does not need all of the conditions (24a), (24b), (24c), and (24d) to be satisfied for FD. By analyzing these conditions, we can conclude that

- Although (24a) can guarantee that the SE-error vector will be independent of the states of (3), it is always possible to obtain the values of $z_{k}$ during operation. Thus, it does not require the satisfaction of (24a) to avoid the effect of $z_{k}$ in FD.

- Equation (24b) is related to the system outputs. The satisfaction of (24b) can directly make the SE-error vector independent of $y_{k}$. Because $y_{k}$ is measurable, we can retain it in the dynamics of $e_{k}$, which means that the satisfaction of (24b) can be unnecessary as well.

- Equation (24c) is the parametric matrix of the system inputs. Similar to the outputs, because the inputs can be obtained, we can treat (24c) as a design degree of freedom as well.

- Equation (24d) is the decoupling condition of the first group of unknown inputs. If (24d) is satisfied, the effect of $\omega_{1, k}^{i}$ on robust FD can be removed producing tighter residual sets and consequently reducing the conservatism of FD. Otherwise, the proposed SUIO-based approach will degenerate to a traditional set-based passive robust FD approach.

Thus, by removing (24a) or (24b) or (24c) or part/all of them, it would be able to obtain more design degrees of freedom for the parametric matrices of UIO when satisfying the design conditions for the implementation of robust SE and FD.

4.3.2. Extended SUIO-based fault detection. It can be observed that, if (24a) or (24b) or (24c) or a combination of them are not satisfied, the unknown inputs will indirectly affect $e_{k}^{x}$ by means of $z_{k}$, $y_{k}$, or $u_{k}$. In this case, (3) is not a UIO or even an observer of system (1). 
Without loss of generality, we only consider the case that all (24a), (24b), and (24c) are not satisfied for illustrative purposes. Thus, under the condition $(24 \mathrm{~d})$, the dynamics of the SE-error vector can be obtained as

$$
\begin{aligned}
e_{k+1}^{x}= & \sum_{i=1}^{N} \lambda_{i}\left(\rho_{k}\right)\left\{\Lambda_{i, 0} e_{k}^{x}+\Lambda_{i, 1} z_{k}+\Lambda_{i, 2} y_{k}+\Lambda_{i, 3} u_{k}\right. \\
& \left.+\Lambda_{i, 8} \omega_{2, k}+\Lambda_{i, 9} \omega_{3, k}+\Lambda_{i, 6} \eta_{k}\right\}+\Lambda_{i, 5} \eta_{k+1} .
\end{aligned}
$$

By considering the sets of $\omega_{2, k}, \omega_{3, k}, \eta_{k+1}$, and $\eta_{k}$ in (34), the set of the SE-error vector is obtained as

$$
\begin{aligned}
\mathbb{E}_{k+1}^{x}= & \left.\sum_{i=1}^{N} \lambda_{i}\left(\rho_{k}\right) \Lambda_{i, 0}\right) \mathbb{E}_{k}^{x} \oplus\left\{\left(\sum_{i=1}^{N} \lambda_{i}\left(\rho_{k}\right) \Lambda_{i, 1}\right) z_{k}\right\} \oplus\left\{\left(\sum_{i=1}^{N} \lambda_{i}\left(\rho_{k}\right) \Lambda_{i, 2}\right) y_{k}\right\} \\
& \left.\oplus\left\{\left(\sum_{i=1}^{N} \lambda_{i}\left(\rho_{k}\right) \Lambda_{i, 3}\right) u_{k}\right\} \oplus \sum_{i=1}^{N} \lambda_{i}\left(\rho_{k}\right) \Lambda_{i, 8}\right) W_{2} \\
& \left.\left.\oplus \sum_{i=1}^{N} \lambda_{i}\left(\rho_{k}\right) \Lambda_{i, 9}\right) W_{3} \oplus \Lambda_{i, 5} V \oplus \sum_{i=1}^{N} \lambda_{i}\left(\rho_{k}\right) \Lambda_{i, 6}\right) V .
\end{aligned}
$$

Moreover, the augmented dynamics of (3a) and (34) is obtained as

$$
\begin{aligned}
\zeta_{k+1}= & \sum_{i=1}^{N} \lambda_{i}\left(\rho_{k}\right)\left\{\Phi_{i, 0} \zeta_{k}+\Phi_{i, 1} y_{k}+\Phi_{i, 2} u_{k}+\Phi_{i, 3} \omega_{2, k}\right. \\
& \left.+\Phi_{i, 4} \omega_{3, k}+\Phi_{i, 5} \eta_{k}\right\}+\Phi_{i, 6} \eta_{k+1},
\end{aligned}
$$

where

$$
\begin{gathered}
\zeta_{k}=\left[\begin{array}{c}
z_{k} \\
e_{k}^{x}
\end{array}\right], \Phi_{i, 0}=\left[\begin{array}{cc}
N_{i} & \mathbf{0} \\
\Lambda_{i, 1} & \Lambda_{i, 0}
\end{array}\right], \Phi_{i, 1}=\left[\begin{array}{c}
K_{i} \\
\Lambda_{i, 2}
\end{array}\right], \Phi_{i, 2}=\left[\begin{array}{c}
T_{i} \\
\Lambda_{i, 3}
\end{array}\right], \\
\Phi_{i, 3}=\left[\begin{array}{c}
\mathbf{0} \\
\Lambda_{i, 8}
\end{array}\right], \Phi_{i, 4}=\left[\begin{array}{c}
\mathbf{0} \\
\Lambda_{i, 9}
\end{array}\right], \Phi_{i, 5}=\left[\begin{array}{c}
\mathbf{0} \\
\Lambda_{i, 6}
\end{array}\right], \Phi_{i, 6}=\left[\begin{array}{c}
\mathbf{0} \\
\Lambda_{i, 5}
\end{array}\right] .
\end{gathered}
$$

Theorem 4.1

The dynamics (36) is a state observer for the system (1) if and only if there exist a symmetric positive definite matrix $P$ and parametric matrices $N_{i}, M, K_{i}, H$ such that

$$
\Psi_{i, j}=\left[\begin{array}{ccc}
\Phi_{i, 0}^{T} P \Phi_{j, 0}-P & \Phi_{i, 0}^{T} P \Phi_{j, 1} & \Phi_{i, 0}^{T} P \Phi_{j, 2} \\
* & \Phi_{i, 1}^{T} P \Phi_{j, 1} & \Phi_{i, 1}^{T} P \Phi_{j, 2} \\
* & * & \Phi_{i, 2}^{T} P \Phi_{j, 2},
\end{array}\right]<\mathbf{0}
$$

for all $i \in \mathbb{I}$ and $j \in \mathbb{I}$, where $*$ denotes the symmetric elements of the symmetric matrix $\Psi_{i, j}$. Moreover, a robust SE set at time instant $k$ can be obtained as

$$
\hat{X}_{k}=\left\{\hat{x}_{k}\right\} \oplus \mathbb{E}_{k}^{x}
$$

where $\hat{x}_{k}$ and $\mathbb{E}_{k}^{x}$ are obtained from (3) and (35), respectively, and any point inside the set $\hat{X}_{k}$ can be used as a SE of the system (1).

Proof

We define the following Lyapunov function:

$$
V\left(\zeta_{k}\right)=\zeta_{k}^{T} P \zeta_{k}
$$


Considering that $\omega_{2, k}, \omega_{3, k}, \eta_{k}$, and $\eta_{k+1}$ are generally bounded unknown inputs, disturbances, and measurement noises, when analyzing the asymptotic stability, we assume that they are zero. In this way, it can be obtained that

$$
\begin{aligned}
\Delta V\left(\zeta_{k}\right)= & \zeta_{k+1}^{T} P \zeta_{k+1}-\zeta_{k}^{T} P \zeta_{k} \\
= & \sum_{i=1}^{N} \sum_{j=1}^{N} \lambda_{i}\left(\rho_{k}\right) \lambda_{j}\left(\rho_{k}\right)\left\{\zeta_{k}^{T}\left(\Phi_{i, 0}^{T} P \Phi_{j, 0}-P\right) \zeta_{k}+y_{k}^{T} \Phi_{i, 1}^{T} P \Phi_{j, 1} y_{k}\right. \\
& \left.+u_{k}^{T} \Phi_{i, 2}^{T} P \Phi_{j, 2} u_{k}+2 \zeta_{k}^{T} \Phi_{i, 0}^{T} P \Phi_{j, 1} y_{k}+2 \zeta_{k}^{T} \Phi_{i, 0}^{T} P \Phi_{j, 2} u_{k}+2 y_{k}^{T} \Phi_{i, 1}^{T} P \Phi_{j, 2} u_{k}\right\} \\
= & \sum_{i=1}^{N} \sum_{j=1}^{N} \lambda_{i}\left(\rho_{k}\right) \lambda_{j}\left(\rho_{k}\right)\left[\begin{array}{lll}
\zeta_{k}^{T} & y_{k}^{T} & u_{k}^{T}
\end{array}\right] \Psi_{i, j}\left[\begin{array}{l}
\zeta_{k} \\
y_{k} \\
u_{k}
\end{array}\right]
\end{aligned}
$$

with

$$
\Psi_{i, j}=\left[\begin{array}{ccc}
\Phi_{i, 0}^{T} P \Phi_{j, 0}-P & \Phi_{i, 0}^{T} P \Phi_{j, 1} & \Phi_{i, 0}^{T} P \Phi_{j, 2} \\
* & \Phi_{i, 1}^{T} P \Phi_{j, 1} & \Phi_{i, 1}^{T} P \Phi_{j, 2} \\
* & * & \Phi_{i, 2}^{T} P \Phi_{j, 2},
\end{array}\right]
$$

Thus, under the condition that the matrices $\Psi_{i, j}$ for all $i, j=\in \mathbb{I}$ are negative definite, that is,

$$
\Psi_{i, j}<\mathbf{0} \text {, for } i, j \in \mathbb{I},
$$

it can be guaranteed that

$$
\Delta V\left(\zeta_{k}\right)<0
$$

which implies the asymptotic stability of the dynamics (36).

Furthermore, according to (4), $x=\hat{x}_{k}+e_{k}^{x}$. By further considering the set of $e_{k}^{x}$ in (35), a robust SE set can be computed as

$$
\hat{X}_{k}=\left\{\hat{x}_{k}\right\} \oplus \mathbb{E}_{k}^{x}
$$

Thus, the proof is complete.

\section{Remark 4.4}

Theorem 4.1 is only a sufficient condition to guarantee the asymptotic stability of the observer. Thus, if the parametric matrices of the observer can satisfy Theorem 4.1, the observer stability can be guaranteed. However, if Theorem 4.1 is not satisfied, it does not imply that there do not exist parametric matrices to design a stable observer. This means that as long as we can find parametric matrices able to stabilize (36), the proposed approach can be used for robust SE and FD even though the designed parametric matrices do not satisfy Theorem 4.1. Additionally, if we consider all the parametric matrices of the UIO as designed parameters, (38) is not an LMI. However, in order to solve (38), we could first assign part of parametric matrices of (3) to be known values and then compute the other parametric matrices. In this way, (38) can be transformed into an LMI for the reduction of computational complexity.

Similarly, the sets of the residual vector can be computed by using (29) and (35) as well. Finally, the FD task can be carried out in real time by means of the same criterion (30b).

\section{Remark 4.5}

In the case that (24a) or (24b) or (24c) or a combination out of these three conditions are not satisfied, we can take the same measures to do robust FD. Notice that all FD steps are the same, while the difference only consists in modifying (34) and (35) according to the corresponding cases. 
4.3.3. More discussions regarding the SUIO-based framework. In Section 4.3.2, the FD task can be performed as long as the parametric matrices of (3) can satisfy Proposition 4.1 and the condition (24d), which are less conservative than the solutions given in Sections 3 and 4.

Actually, all the solutions aforementioned are affected by the measurement noises at two consecutive time instants, that is, $\eta_{k}$ and $\eta_{k+1}$. Thus, when the set theory is applied for robust FD, we have to consider the set $V$ two times as in (35), which increases the FD conservatism a lot. Thus, if we can remove the measurement noise vector from (34), the FD performance will be improved at the price of complicating design conditions of the observer described in Theorem 4.1. In order to clearly explain this problem, the following cases are considered:

- The observer described in Theorem 4.1 can simultaneously satisfy the extra conditions

$$
\begin{aligned}
& \Lambda_{i, 5}=\mathbf{0}, \\
& \Lambda_{i, 6}=\mathbf{0} .
\end{aligned}
$$

Although, in this situation, we could remove both sets $V$ in (35) (i.e., the proposed approach will have the least FD conservatism), it is quite difficult (even impossible for most of systems) to find a parametric matrix $H$ that simultaneously satisfies (43a) and (43b) for all $i \in \mathbb{I}$.

- The observer described in Theorem 4.1 is only designed to satisfy one extra condition (43b). In this case, we can first design $H$ according to (24d). Then, we need to find a pair of $M$ and $K_{i, 1}$ that can simultaneously satisfy the condition (43b) and the stability condition of observer together with the matrices $H$ and $N_{i}$ in Theorem 4.1.

\section{Lemma 4.1}

The condition for the equation $X Q=R$ is that $R Q^{\{1\}} Q=R$ can hold, where $Q$ and $R$ are known and compatible matrices. The general form of solutions of the equation is

$$
X=R Q^{\{1\}}+Y\left(I-Q Q^{\{1\}}\right)
$$

where $Y$ is an arbitrary matrix with proper dimensions, $I$ is the identity matrix with proper dimensions, and $Q^{\{1\}}$ denotes the $\{1\}$ pseudo inverse of the matrix $Q$.

According to Lemma 4.1, the following results can be used to compute the multiplication of $M$ and $K_{i, 1}$, that is,

$$
M K_{i, 1}=Y\left(I-F F^{\{1\}}\right)
$$

where $Y$ and $I$ are defined as in Lemma 4.1. In order to obtain $M$ and $K_{i, 1}$, respectively, we should first choose a value for $M$ (or $K_{i, 1}$ ) and determine a value for $Y$. Then, the matrix $K_{i, 1}$ (or $M$ ) can be obtained by solving (44).

\section{Proposition 4.1}

The necessary and sufficient condition for (44) to have solutions is

$$
\operatorname{rank}(M)=\operatorname{rank}\left(Y\left(I-F F^{\{1\}}\right)\right)
$$

or

$$
\operatorname{rank}\left(K_{i, 1}\right)=\operatorname{rank}\left(Y\left(I-F F^{\{1\}}\right)\right)
$$

when $K_{i, 1}$ (or $M$ ) is chosen as the unknown matrix of (44).

Proof

The proof is straightforward and omitted here. 
Remark 4.6

It can be observed that there exist enough degrees of freedom in the design of the observer proposed in Theorem 4.1 when choosing $Y$ and $M$ (or $K_{i, 1}$ ) to guarantee that (44) is solvable and all design conditions can be satisfied. Note that these additional design degrees of freedom represent one of the main contributions of the current paper with respect to the existing UIO-based and the set-based robust SE and FD estimation approaches.

Thus, under the condition (43b), the dynamics (35) can be further reduced to

$$
\begin{aligned}
\mathbb{E}_{k+1}^{x}= & \left.\sum_{i=1}^{N} \lambda_{i}\left(\rho_{k}\right) \Lambda_{i, 0}\right) \mathbb{E}_{k}^{x} \oplus\left\{\left(\sum_{i=1}^{N} \lambda_{i}\left(\rho_{k}\right) \Lambda_{i, 1}\right) z_{k}\right\} \\
& \oplus\left\{\left(\sum_{i=1}^{N} \lambda_{i}\left(\rho_{k}\right) \Lambda_{i, 2}\right) y_{k}\right\} \oplus\left\{\left(\sum_{i=1}^{N} \lambda_{i}\left(\rho_{k}\right) \Lambda_{i, 3}\right) u_{k}\right\} \\
& \left.\left.\oplus \sum_{i=1}^{N} \lambda_{i}\left(\rho_{k}\right) \Lambda_{i, 8}\right) W_{2} \oplus \sum_{i=1}^{N} \lambda_{i}\left(\rho_{k}\right) \Lambda_{i, 9}\right) W_{3} \oplus \Lambda_{i, 5} V .
\end{aligned}
$$

In a similar way, we can use (29) to compute the residual sets corresponding to (47) and the FD task can be further implemented by means of the FD criterion (30b) and (31).

\section{ILLUSTRATIVE EXAMPLES}

We will use two examples (a vehicle and a continuous stirred tank reactor (CSTR)) well-accepted in the control community to illustrate the proposed approach. Without loss of generality, the effectiveness of the proposed SUIO approach will be shown only on the basis of the least conservative results obtained in (47).

\subsection{Case study 1}

The vehicle LPV model taken from [23] is used as the first case study. The input is the steering angle $u_{L}$, the states are the slide slip angle $\beta$ and the yaw rate $\dot{\psi}$, and the outputs are also the slide slip angle $\beta$ and the yaw rate $\dot{\psi}$ in this example. We consider the wind force as an unknown input perturbation signal $F_{w}(t)$. Besides, we further define two unknown inputs to describe the modeling errors and generally denote other unknown factors that affect the system, respectively. $\eta_{k}$ includes the measurement noises of the slide slip angle $\beta$ and the yaw rate $\dot{\psi}$. Thus, we have three unknown inputs needed to deal with in this example. The nonlinear model of the vehicle can be written as

$$
\begin{gathered}
{\left[\begin{array}{l}
\dot{\beta}(t) \\
\ddot{\psi}(t)
\end{array}\right]=\left[\begin{array}{cc}
-\frac{c_{r}+c_{f}}{m v(t)} & \frac{c_{r} l_{r}-c_{f} l_{f}}{m v^{2}(t)}-1 \\
\frac{c_{r} l_{r}-c_{f} l_{f}}{I_{z}} & -\frac{c_{r} l_{f}^{2}+c_{f} l_{f}^{2}}{I_{z} v(t)}
\end{array}\right]\left[\begin{array}{l}
\beta(t) \\
\dot{\psi}(t)
\end{array}\right]+\left[\begin{array}{c}
\frac{c_{f}}{m v(t)} \\
\frac{c_{r} l_{f}}{I_{z}}
\end{array}\right] u_{L}(t)+E_{\omega} \omega(t),} \\
y(t)=\left[\begin{array}{ll}
1 & 0 \\
0 & 1
\end{array}\right]\left[\begin{array}{l}
\beta(t) \\
\dot{\psi}(t)
\end{array}\right]+F_{\eta} \eta(t),
\end{gathered}
$$

where $\omega(t)$ includes the three unknown inputs, $E_{\omega}$ is its corresponding parametric matrix, $\eta(t)$ denotes the measurement noises, $F_{\eta}$ is the corresponding parametric matrix, and the definitions and values of all the other parameters appearing in (48) can be found in [24].

We define two scheduling variables $\rho_{1, k}=\frac{l}{v}$ and $\rho_{2, k}=\frac{l}{v^{2}}$. On the basis of the two scheduling variables, the nonlinear model (48) can be equivalently transformed into an LPV model as in (1). In this example, the speed $v(t)$ varies between 20 and $40 \mathrm{~km} / \mathrm{h}$. Because $v(t)$ is bounded, we can know that $\rho_{1, k}$ and $\rho_{2, k}$ are also bounded. This implies that a polytope bounding the vector composed of these two scheduling variables can be obtained and it has four vertices. Moreover, by using the vertices of this polytope, the vehicle model can be transformed into a polytopic LPV form. However, 
because $\rho_{1, k}$ and $\rho_{2, k}$ have a definite mathematical relationship, the number of the independent vertices of the LPV model can be reduced to three [23]. Note that, in this paper, the LPV model (48) is discretized with a sampling time of $0.01 \mathrm{~s}$, whose corresponding parametric matrices are obtained as

$$
\begin{aligned}
& A_{1}=\left[\begin{array}{cc}
0.9194 & -0.0078 \\
0.1729 & 0.9188
\end{array}\right], A_{2}=\left[\begin{array}{cc}
0.8388 & -0.0078 \\
0.1729 & 0.8375
\end{array}\right], A_{3}=\left[\begin{array}{cc}
0.8388 & -0.0011 \\
0.1729 & 0.8375
\end{array}\right], \\
& F=\left[\begin{array}{cc}
0.01 & 0.03 \\
0.004 & 0.015
\end{array}\right], B_{1}=\left[\begin{array}{l}
0.0378 \\
0.2994
\end{array}\right], B_{2}=\left[\begin{array}{l}
0.0756 \\
0.2994
\end{array}\right], B_{3}=\left[\begin{array}{l}
0.0756 \\
0.2994
\end{array}\right], \\
& E_{1}=\left[\begin{array}{lll}
0.004 & 0.0005 & 0.0009 \\
0.008 & 0.0003 & 0.0254
\end{array}\right], E_{2}=\left[\begin{array}{lll}
0.004 & 0.0005 & 0.0018 \\
0.008 & 0.0003 & 0.0254
\end{array}\right] \text {, } \\
& E_{3}=\left[\begin{array}{lll}
0.004 & 0.0005 & 0.0018 \\
0.008 & 0.0003 & 0.0254
\end{array}\right] \text {, }
\end{aligned}
$$

where the notations $E_{1}, E_{2}, E_{3}$, and $F$ have the same meanings as defined previously in the paper.

According to the proposed approach, the unknown inputs should be divided into three groups, where the first group is actively decoupled, while the remaining unknown inputs are passively decoupled. Thus, without loss of generality, for three groups of vertices, we only present their corresponding first group of unknown inputs, that is,

$$
E_{1,1}=\left[\begin{array}{l}
0.004 \\
0.008
\end{array}\right], E_{2,1}=\left[\begin{array}{l}
0.004 \\
0.008
\end{array}\right], E_{3,1}=\left[\begin{array}{l}
0.004 \\
0.008
\end{array}\right] .
$$

\section{Remark 5.1}

In this example, it is obvious that the signals $\omega(t)$ and $\eta(t)$ and their parametric matrices $E_{\omega}$ and $F_{\eta}$ are difficult to be obtained exactly. If we want to know them, plenty of experiments are needed in reality. However, considering that the main purpose of the current paper is only to propose a methodology instead of a real application, without loss of generality, we only select a group of reasonable values of the bounds of these signals and the corresponding parametric matrices empirically based on a large number of simulations for the illustration of the proposed methodology.

By using the aforementioned data and the proposed approach, a stable UIO for the LPV system can be designed, whose tuning parameters to guarantee its stability and performance of robust FD and estimations for the illustration can be obtained as

$$
\begin{aligned}
& H=\left[\begin{array}{ll}
0.2411 & 0.3795 \\
0.3876 & 0.8062
\end{array}\right], M=\left[\begin{array}{ll}
0.9418 & 0.8291 \\
0.0172 & 0.6266
\end{array}\right], \\
& N_{1}=\left[\begin{array}{cc}
0.84 & 0 \\
0 & 0.57
\end{array}\right], N_{2}=\left[\begin{array}{cc}
0.48 & 0 \\
0 & 0.75
\end{array}\right], N_{3}=\left[\begin{array}{cc}
0.84 & 0 \\
0 & 0.59
\end{array}\right] \text {, } \\
& T_{1}=\left[\begin{array}{l}
0.8776 \\
0.0144
\end{array}\right], T_{2}=\left[\begin{array}{l}
0.5811 \\
0.6372
\end{array}\right], T_{3}=\left[\begin{array}{l}
0.5289 \\
0.6944
\end{array}\right] \text {, } \\
& K_{1,1}=\left[\begin{array}{ll}
0 & 0 \\
0 & 0
\end{array}\right], K_{1,2}=\left[\begin{array}{ll}
0.5387 & 0.7266 \\
0.6505 & 0.0945
\end{array}\right], K_{2,1}=\left[\begin{array}{ll}
0 & 0 \\
0 & 0
\end{array}\right] \text {, } \\
& K_{2,2}=\left[\begin{array}{ll}
0.2943 & 0.9263 \\
0.1799 & 0.0682
\end{array}\right], K_{3,1}=\left[\begin{array}{ll}
0 & 0 \\
0 & 0
\end{array}\right], K_{3,2}=\left[\begin{array}{cc}
0.65130 .056 & \\
0.8646 & 0.8169
\end{array}\right] \text {. }
\end{aligned}
$$

\section{Remark 5.2}

In order to obtain satisfactory performance, properly tuning the parametric matrices of the SUIO under the aforementioned rank-based design conditions of UIO is the key to guarantee the stability (or under Theorem 4.1 for stability) of the SUIO for robust SE and FD.

Moreover, the sets of the unknown inputs and the measurement noises obtained according to Remark 5.1 are given as zonotopes: 


$$
W=\left[\begin{array}{l}
0 \\
0 \\
0
\end{array}\right] \oplus\left[\begin{array}{ccc}
0.01 & 0 & 0 \\
0 & 0.01 & 0 \\
0 & 0 & 0.01
\end{array}\right] \mathbb{B}^{3}, \quad V=\left[\begin{array}{l}
0 \\
0
\end{array}\right] \oplus\left[\begin{array}{cc}
0.1 & 0 \\
0 & 0.1
\end{array}\right] \mathbb{B}^{2}
$$

In this example, without loss of generality, we consider that a fault in the actuator with the following feature has occurred in the system, that is,

$$
f=0.1+0.2 \text { rand }
$$

where rand denotes a random value inside the interval $[0,1]$. It is assumed that the fault occurs at time instant $k=40$ and the detection of the fault is shown in Figure 1.

Note that $R_{k}(1)$ and $R_{k}(2)$ denote the first and second components of residual zonotopes. In Figure 1, as defined, the fault occurs at time instant $k=40$, while we can observe that $0 \notin R_{41}(2)$, which indicates that the fault has been detected at time instant $k=41$ and illustrates the effectiveness of the proposed FD approach. Moreover, $\hat{X}_{k}(1)$ and $\hat{X}_{k}(2)$ are the two components of the robust SE zonotope $\hat{X}_{k}$ in magenta as shown in Figure 2 . The SEs $\hat{x}_{k}^{e}(1)$ and $\hat{x}_{k}^{e}(2)$ in blue lines are taken as the centers of $\hat{X}_{k}(1)$ and $\hat{X}_{k}(2)$, respectively, where it can be observed that, before the fault, the proposed observer can provide satisfactory SEs for the system, while after the fault $(k>40)$, because the change of system model induced by the fault, the quality of SEs becomes worse. This illustrates the effectiveness of SE of the proposed observer.

\subsection{Case study 2}

The CSTR system is used as the second case study to further illustrate the effectiveness of the proposed approach. The details and nonlinear model of the CSTR system used in this paper can be
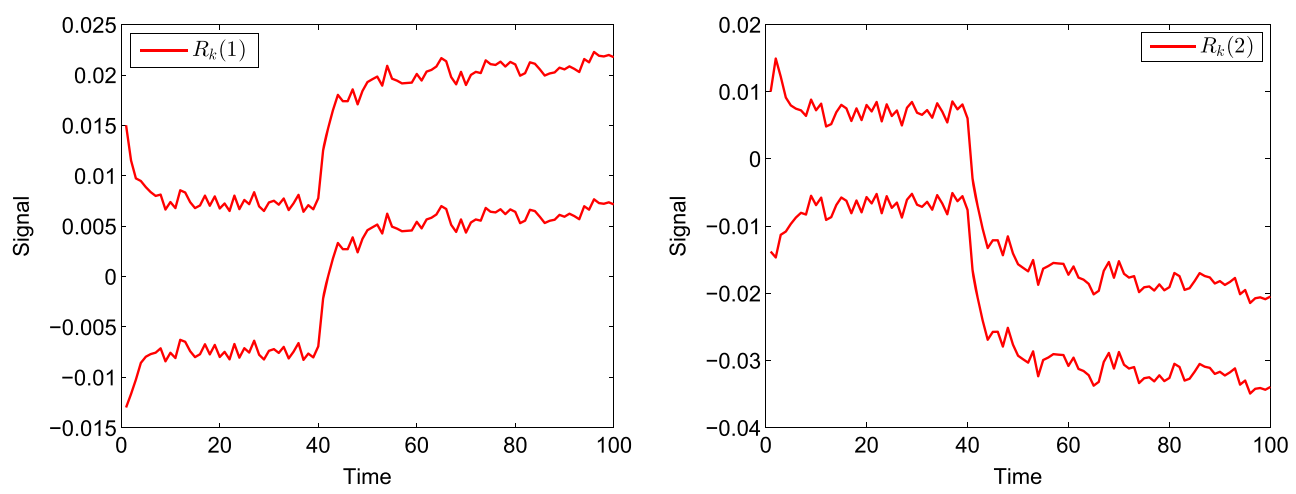

Figure 1. FD of Vehicle. [Colour figure can be viewed at wileyonlinelibrary.com]
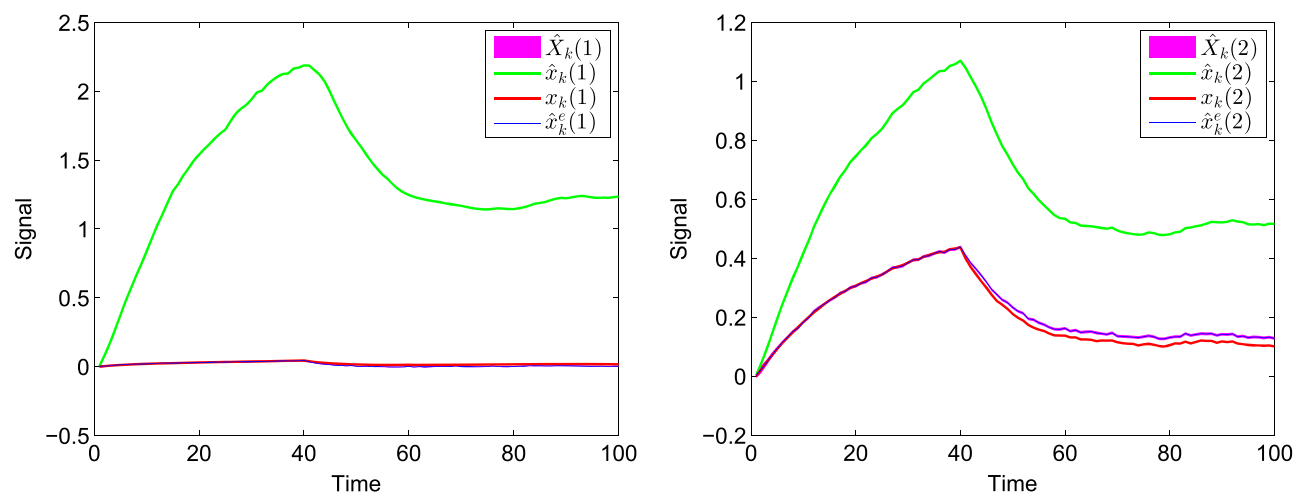

Figure 2. State Estimation of Vehicle. [Colour figure can be viewed at wileyonlinelibrary.com] 
found in [25], where the conversion rate of reaction and the temperature of reactor are defined as two system states and two outputs. Thus, the output matrix can be first obtained as

$$
C=\left[\begin{array}{ll}
1 & 0 \\
0 & 1
\end{array}\right] \text {. }
$$

In [26], the nonlinear model of the CSTR system was transformed into a polytopic LPV model with the form in (1). Note that all the parameters of the LPV model used in the current paper can be found in [26]. Thus, more details can be recalled there as well. Three vertices of each polytope corresponding to each parametric matrix of the LPV model taken from [26] are presented as

$$
\begin{aligned}
& A_{1}=\left[\begin{array}{cc}
0.75 & 0.0019 \\
-0.2238 & 0.8262
\end{array}\right], A_{2}=\left[\begin{array}{cc}
0.6203 & 0.0762 \\
-1.2337 & 1.3265
\end{array}\right], \\
& A_{3}=\left[\begin{array}{cc}
0.3068 & 0.0442 \\
-3.6621 & 1.0765
\end{array}\right], F=\left[\begin{array}{ll}
0.015 & 0.023 \\
0.034 & 0.025
\end{array}\right], \\
& B_{1}=\left[\begin{array}{l}
0.0004 \\
0.0546
\end{array}\right], B_{2}=\left[\begin{array}{l}
0.0023 \\
0.0698
\end{array}\right], B_{3}=\left[\begin{array}{l}
0.0015 \\
0.0634
\end{array}\right] .
\end{aligned}
$$

It is known that there always exist modeling errors, discretizing errors, and linearizing errors. Thus, we use three unknown inputs to model these errors, respectively, and the coefficient matrices of unknown inputs for the three groups of vertices are given as

$$
E_{1}=E_{2}=E_{2}=\left[\begin{array}{ccc}
0.03 & 0.007 & 0.032 \\
0.09 & 0.004 & 0.0241
\end{array}\right]
$$

The unknown inputs and measurement noises are bounded by the following zonotopes:

$$
W=\left[\begin{array}{l}
0 \\
0 \\
0
\end{array}\right] \oplus\left[\begin{array}{ccc}
0.1 & 0 & 0 \\
0 & 0.1 & 0 \\
0 & 0 & 0.1
\end{array}\right] \mathbb{B}^{3}, V=\left[\begin{array}{l}
0 \\
0
\end{array}\right] \oplus\left[\begin{array}{cc}
0.1 & 0 \\
0 & 0.1
\end{array}\right] \mathbb{B}^{2} .
$$

For all the three groups of vertices, the first unknown input is actively decoupled for the purpose of robust FD. As a result, it is obtained that

$$
E_{1,1}=E_{2,1}=E_{3,1}=\left[\begin{array}{l}
0.03 \\
0.09
\end{array}\right]
$$

\section{Remark 5.3}

The notations of the parameters appearing in the previous discussion in this case study fully respect the definitions of parameters in the model (1). Additionally, the values of bounds of the unknown inputs and the measurement noises and their corresponding parametric matrices $E$ and $F$ are obtained in the same way as case study 1 empirically based on a large number of simulations (Remark 5.1).

Based on the aforementioned information, the parametric matrices of a stable UIO designed for the LPV system can be obtained as

$$
\begin{aligned}
H & =\left[\begin{array}{l}
0.08740 .3042 \\
0.66080 .7797
\end{array}\right], M=\left[\begin{array}{cc}
0.299 & 0.0558 \\
0.4188 & 0.0309
\end{array}\right], \\
N_{1} & =\left[\begin{array}{cc}
0.73 & 0 \\
0 & 0.45
\end{array}\right], N_{2}=\left[\begin{array}{cc}
0.36 & 0 \\
0 & 0.95
\end{array}\right], N_{3}=\left[\begin{array}{cc}
0.73 & 0 \\
0 & 0.64
\end{array}\right], \\
T_{1} & =\left[\begin{array}{l}
0.0499 \\
0.1013
\end{array}\right], T_{2}=\left[\begin{array}{l}
0.3776 \\
0.9516
\end{array}\right], T_{3}=\left[\begin{array}{l}
0.3911 \\
0.2233
\end{array}\right],
\end{aligned}
$$



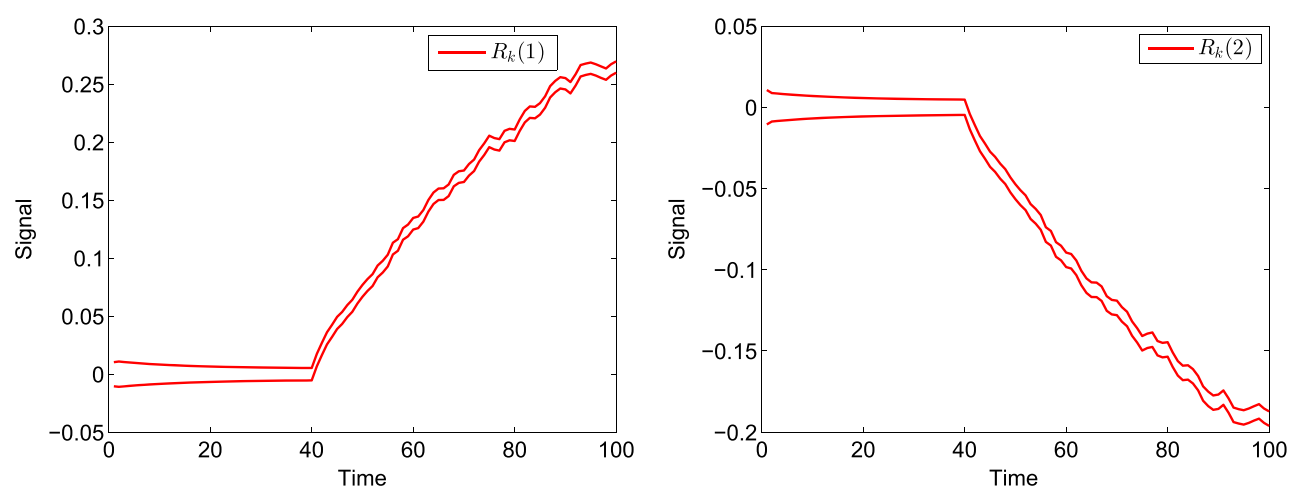

Figure 3. FD of CSTR. [Colour figure can be viewed at wileyonlinelibrary.com]
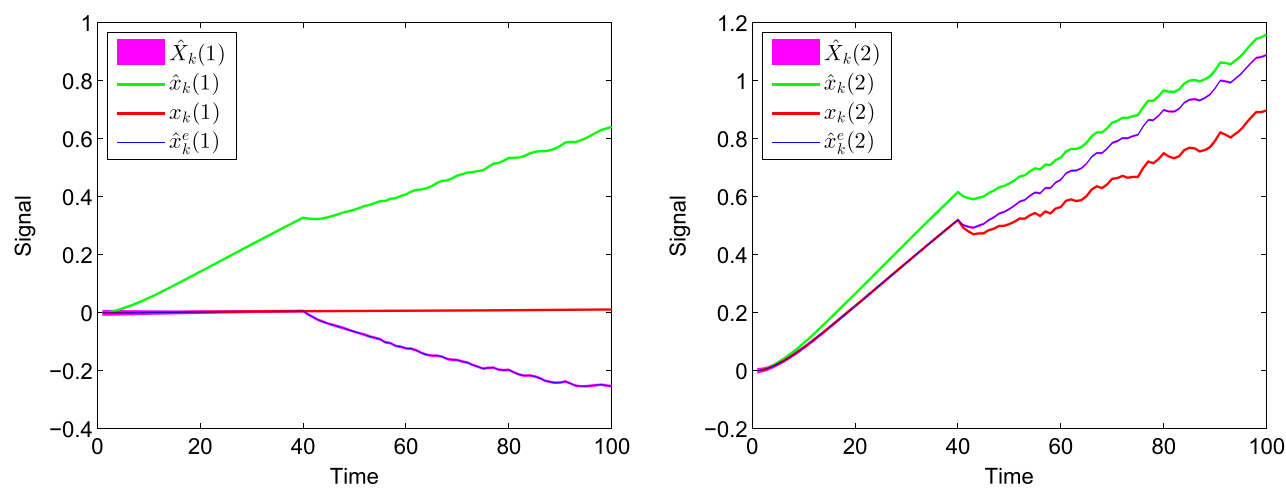

Figure 4. State Estimation of CSTR. [Colour figure can be viewed at wileyonlinelibrary.com]

$$
\begin{aligned}
K_{1,1} & =\left[\begin{array}{ll}
0 & 0 \\
0 & 0
\end{array}\right], K_{1,2}=\left[\begin{array}{ll}
0.1866 & 0.0662 \\
0.2709 & 0.3335
\end{array}\right], K_{2,1}=\left[\begin{array}{ll}
0 & 0 \\
0 & 0
\end{array}\right], \\
K_{2,2} & =\left[\begin{array}{ll}
0.0719 & 0.3118 \\
0.9045 & 0.5918
\end{array}\right], K_{3,1}=\left[\begin{array}{ll}
0 & 0 \\
0 & 0
\end{array}\right], K_{3,2}=\left[\begin{array}{lll}
0.7424 & 0.5989 \\
0.8871 & 0.6896
\end{array}\right] .
\end{aligned}
$$

Similarly, in order to illustrate the proposed approach, we define a fault scenario that the simulation time is 100 time instants and an actuator fault $f=0.6+0.2$ rand occurs at time instant $k=40$. Then, the UIO is used to monitor the CSTR system; the results of robust FD are shown in Figure 3. It can be seen that after the fault occurs at time instant $k=40$, it is detected that $0 \notin R_{41}(1)$ and $0 \notin R_{41}(2)$, which indicates that the fault has been detected at time instant $k=41$. Moreover, $\hat{X}_{k}(1)$ and $\hat{X}_{k}(2)$ are the two components of the robust SE zonotope $\hat{X}_{k}$ in magenta as shown in Figure 4. The SEs $\hat{x}_{k}^{e}(1)$ and $\hat{x}_{k}^{e}(2)$ in blue lines are taken as the centers of $\hat{X}_{k}(1)_{k}$ and $\hat{X}_{k}(2)$, respectively, where before the fault, the SEs can well approach the real states in red lines, while after the fault, the SEs become worse. These results illustrate the effectiveness of the proposed approach.

\section{CONCLUSIONS}

This paper has proposed a robust FD approach for the LPV system by integrating the UIO and the set theory. The proposed SUIO-based approach has inherited the advantages of both the traditional UIObased approaches and the set-based approaches. On the one hand, the design conditions of the SUIO are less restrictive than those of the traditional UIO when applied to robust FD and SE. On the other hand, the proposed method can decouple not only the effect of part of unknown inputs with respect to the traditional set-based approaches but also the effect of the measurement noises with respect to the 
traditional UIO-based approaches. Thus, in this sense, the proposed method have advantages over both the traditional set-based and UIO-based approaches on the conservatism of FD conditions and design conditions. Moreover, as explained in the contents, the proposed SUIO-based FD framework have sufficient design degrees of freedom when different features and performance are required by a monitored system. Additionally, another important point is that we have established a group of sufficient conditions using matrix inequalities for the stability of SUIO. In the future research, we will continue to study how to apply the proposed method to fault isolation and fault-tolerant control of the LPV system.

\section{ACKNOWLEDGEMENTS}

This work was supported by the Natural Science Foundation of Guangdong (no. 2015A030313881), the National Natural Science Foundation of China (no. 61673239), the Shenzhen Key Lab of Space Robotic Technology and Telescience, the Spanish Government (MINECO) through the project CICYT ECOCIS (ref. DPI2013-48243-C2-1-R), and MINECO and FEDER through the project CICYT HARCRICS (ref. DPI2014-58104-R).

\section{REFERENCES}

1. Blanke M, Kinnaert M, Lunze J, Staroswiecki M. Diagnosis and Fault-tolerant Control. Springer-Verlag: Berlin, Germany, 2006.

2. Puig V. Fault diagnosis and fault tolerant control using set-membership approaches: Application to real case studies. International Journal of Applied Mathematics and Computer Science 2010; 20(4):619-635.

3. Blesa J, Puig V, Romera J, Saludes J. Fault diagnosis of wind turbines using a set-membership approach. Proceddings of the 18th IFAC World Congress, Milano, Italy, 2011.

4. Rosa P, Silvestre C. Fault detection and isolation of LPV systems using set-valued observers: an application to a fixed-wing aircraft. Control Engineering Practice 2013; 21(3):242 -252.

5. Chen J, Patton RJ, Zhang H-Y. Design of unknown input observers and robust fault detection filters. International Journal of Control 1996; 63(1):85-105.

6. Hui S, Żak SH. Observer design for systems with unknown inputs. International Journal of Applied Mathematics and Computer Science 2005; 15(4):431-446.

7. Kudva P, Viswanadham N, Ramakrishna A. Observers for linear systems with unknown inputs. IEEE Transactions on Automatic Control 1980; 25(1):113-115.

8. Nejjari F, Puig V, Oca SMD, Sadeghzadeh A. Robust fault detection for LPV systems using interval observers and zonotopes. Proceedings of the Joint 48th IEEE Conference on Decision and Control and 28th Chinese Control Conference, Shanghai, P.R.China, December 2009; 1002-1007.

9. Varrier S, Koenig D, Martinez JJ. Robust fault detection for uncertain unknown inputs LPV system. Control Engineering Practice 2014; 22:125 -134.

10. Ichalal D, Mammar S. On unknown input observers for LPV systems. IEEE Transactions on Industrial Electronics 2015; 62(9):5870-5880.

11. Gao Z, Liu X, Chen MZQ. Unknown input observer-based robust fault estimation for systems corrupted by partially decoupled disturbances. IEEE Transactions on Industrial Electronics 2015; 63(4):2537-2547.

12. Guerra P, Puig V, Witczak M. Robust fault detection with unknown-input interval observers using zonotopes. Proceedings of the 17th IFAC World Congress, COEX, Seoul, South Korea, July 2008; 5557-5562.

13. Charandabi BA, Marquez HJ. A novel approach to unknown input filter design for discrete-time linear systems. Automatica 2014; 50:2835-2839.

14. Park TG. Designing fault detection observers for linear systems with mismatched unknown inputs. Journal of Process Control 2013; 23:1016-1022.

15. Millerioux G, Daafouz J. Unknown input observers for switched linear discrete time systems. Proceedings of the 2004 American Control Conference, Boston, United States, July 2004; 5802-5805.

16. Moreno J. Unknown input observers for SISO nonlinear systems. Proceedings of the 39Th IEEE Conference on Decision and Control, Sydney, Austrilia, December 2000; 790-801.

17. Zheng G, Bejarano FJ, Perruquetti W, Richard JP. Unknown input observer for linear time-delay systems. Automatica $2015 ; 61: 35-43$.

18. Wang H, Ju H, Yang G. Fault detection filter design for linear polytopic uncertain continuous-time systems. Acta Automatica Sinica 2010; 36(5):742-750.

19. Patton RJ, Chen J. Observer-based fault detection and isolation: robustness and applications. Control Engineering Practice 1997; 5(5):671 -682.

20. Yang H, Xia Y, Liu B. Fault detection for T-S fuzzy discrete systems in finite-frequency domain. IEEE Transactions on Systems, Man, and CyberneticstPart B: Cybernetics 2011; 41(4):911-920. 
21. Xu F, Tan JB, Liu HD, Puig V, Wang XQ, Liang B, Yuan B. Robust state estimation and fault detection combining unknown input observer and set-membership approach. Proceedings of the 3rd International Conference on Control and Fault-Tolerant Systems, Barcelona, Spain, September 2016; 67-72.

22. Xu F, Tan JB, Wang XQ, Puig V, Liang B. A novel design of unknown input observers using set-theoretic methods for robust fault detection, Boston, USA, July 2016; 5957-5961.

23. Farhat A, Koenig D, Sename O. Fault detection for LPV systems loop shaping H-infinity approach. Proceedings of the First IFAC Workshop on Linear Parameter Varying Systems LPVs 2015, 48(26), Grenoble, France, October 2015; $188-193$.

24. Mammar S, Koenig D. Vehicle handling improvement by active steering. Vehicle System Dynamics 2002; 38(3): 211-242.

25. Cao YY, Frank PM. Analysis and synthesis of nonlinear time-delay systems via fuzzy control approach. IEEE Transactions on Fuzzy Systems 2000; 8(2):200-211.

26. Yang WL, Gao JW, Feng G, Zhang TJ. An optimal approach to output-feedback robust model predictive control of LPV systems with disturbances. International Journal of Robust and Nonlinear Control 2016; 26(15):3253-3273. 